\title{
Problems and Governance Countermeasures of Formation and Development of Uni-cities in China
}

\author{
Zhang Yongjiao \\ Beijing 100101, China; \\ University of Chinese Academy of Sciences, Beijing 100049, China \\ Man Xiaowu*, Ding Hao \\ Gansu Institute of Urban Planning and design, Lanzhou 730000, China
}

Institute of Geographic Sciences and Natural Resources Research (IGSNRR), Chinese Academy of Sciences,

\begin{abstract}
Uni-cities which are located adjacent to each other are the leading regions implementing integration strategy for urban agglomerations development. The aim of this paper is to explore the problems and corresponding governance countermeasures of uni-cities during their formation and development in China, which is of great significance for getting an overall view of urban integration, understanding its rules as well as optimizing urban development pattern in China. This paper uses Qualitative Metasynthesis method to proposes the general spatial distribution pattern of uni-cities which shows a " $17+8+8+4$ " structure firstly. The paper then systematically analyses the main problems of the uni-cities in China, and the results show that the identification standard is lacking, land resource is utilized extensively, and the collaboration is limited, which specifically comprises of limitations of collaborative content, region, and coordinating approach, so the identification standard, development quality and top layer designs are required to be improved and upgraded. Some policy countermeasures and suggestions for the construction of uni-cities are put forward in the end to ensure their sustainable development as well as healthy and orderly evolution to urban agglomerations.
\end{abstract}

KEYWORD: Urban integration; Uni-cities; Pattern; Problems; Countermeasures; China.

\section{INTRODUCTION}

Scholars and city managers of our country proposes the concept of urban integration when researching the interaction between adjacent cities and their trend of integration. Since integration covers extensive meaning and is mostly used as a specific development strategy put forward by government which usually appears in public document and urban plannning text. Researches show that there are different expressions, urban integration or uni-city, but the two represent the same development substance in China. For understanding the process and the corresponding region space, uni-city are refers to the region implementing "urban integration" strategy in this paper. Urban integration means two or more cities which are adjacent to each other, having a sense of geographical identity, admitting their differences and developing complementary function, by convenient transportation and communication infrastructure, seek coordination and consistency in the aspects of economy, society, culture, ecology, space and governance. It is not only a process, but also a state of affairs. Different from regional economic integration which is characterized by economic factors allocation, urban integration is more focused on the spatial integration, equal public service as well as the integration of culture, whose connotation is more specific and means a lot more than economy.

There are many studies concerning regional integration or regional economic integration in academia, while less research has specifically focused on urban integration since it has a strong indigenous meaning. Past relative studies concentrated on typical integrated cites discussing their formation conditions, questions, solutions, practical experiences and strategic conceptions ( $\mathrm{Li}$ et al., 2010; Ge et al., 2012; Xin, 2007; Song, 2008), lacking of systemic pectination and research on a national scale regarding urban integration. Exploring the present spatial distribution pattern, combing the main problems and putting forward corresponding governance countermeasures of urban integration is of great significance for promoting healthy and orderly evolution of urban agglomerations as well as optimizing urban development pattern in china.

\section{THE GENERAL SPATIAL DISTRIBUTION PATTERN OF UNI-CITIES IN CHINA}

Practices of urban integration in China could be traced back to the 1980s. In 1983, Shanghai 
established their planning office of economic district and the integration of Yangtze River Delta began advancing. Besides that, discussions about the integration of Chang-Zhu-Tan golden triangle and Nanchang-Jiujiang industrial corridor has been opened as early as the 1980s. The practices of urban integration develop actively in this century. BeijingTianjin, Shenzhen-Hongkang, Urumchi-Changji, Taiyuan-Yuci, Xi'an-Xianyang integration were proposed during the 10th Five-Year Plan period which are also widely concerned in the academic at present; During the 11th Five-Year Plan period, the uni-cities implementing integration strategy have expanded to Guangzhou-Foshan and ShenzhenDongguan-Huizhou in the Pearl River Delta region, Nanjing-Zhenjiang-Yangzhou, Nanjing-Maanshan in the Yangtze River Delta Region, Shenyang-Fushun,
Zhenzhou-Kaifeng, Hefei-Huainan, WuhanXiaogan, Lanzhou-Baiyin, Changchun-Jilin, and the lateral cities of Yanji-Longjin-Tumen. It is estimated that the cities implementing integration strategy have reached 37 groups by 2014, half of which are launched in the first four years of the 12th Five-Year Plan period. They present a " $17+8+8+4$ " spatial pattern, representing those in the eastern region, central region, western region and northeast region in China (Tab.1; Fig.1), and the distribution is dense in the east and sparse in the west. The Yangtze River Delta, Shandong peninsula, Pearl River Delta and Southern Fujian are ones of the most densely distributed areas of uni-cites. These cities are the leading regions implementing integration strategy developing towards urban agglomerations.

Table 1. The general spatial distribution of uni-cities in China*

\begin{tabular}{|c|c|c|c|}
\hline Area & Uni-cities & Spatial range & $\begin{array}{l}\text { The corresponding } \\
\text { agglomerations }\end{array}$ \\
\hline \multirow{17}{*}{ Eastern region } & Jing-Jin & Beijing, Tianjin & Beijing-Tianjin-Hebei \\
\hline & Guang-Fo & Guangzhou, Foshan & Pearl River Delta \\
\hline & Shen-Guan-Hui & Shezhen, Dongguan, Huizhou & Pearl River Delta \\
\hline & Shen-HK & Shenzhen, Hongkang & Pearl River Delta \\
\hline & $\mathrm{Hu}-\mathrm{Su}-\mathrm{Jia}$ & Shanhai, Suzhou, Jiaxing & Yangtze River Delta \\
\hline & Su-Xi-Chang & Suzhou, Wuxi, Changzhou & Yangtze River Delta \\
\hline & Ning-Zhen-Yang & Nanjing, Zhenjiang, Yangzhou & Yangtze River Delta \\
\hline & Ning-Ma & Nanjing, Maanshan & - \\
\hline & Hang-Jia-Hu-Shao & Hangzhou, Jiaxing, Huzhou, Shaoxing & Yangtze River Delta \\
\hline & Yong-Zhou & Ningbo, Zhoushan & Yangtze River Delta \\
\hline & Ji-Tai-Lai & Jinan, Taian, Laiwu & Shandong peninsula \\
\hline & Ji-Liao & Jinan, Liaocheng & Shandong peninsula \\
\hline & Yan-Wei & Yantai, Weihai & Shandong peninsula \\
\hline & Qing-Wei-Ri & Qingdao, Weifang, Rizhao & Shandong peninsula \\
\hline & Xia-Zhang-Quan & Xiamen, Zhangzhou, Quanzhou & Southern Fujian \\
\hline & Fu-Pu-Ning & Fuzhou, Putian, Ningde & Southern Fujian \\
\hline & Shan-Jie-Chao & Shantou, Jieyang, Chaozhou & Southern Fujian \\
\hline \multirow{8}{*}{ Central region } & Zheng-Bian & Zhengzhou, Kaifeng & Central plain \\
\hline & Tai-Jin & Taiyuan, Jinzhong & Eastern Shanxi \\
\hline & Wu-Xiao & Wuhan, Xiaogan & Mid-Yangtze River \\
\hline & Wu-E-Huang-Huang & Whan, Ezhou, Huangshi, Huanggang & Mid-Yangtze River \\
\hline & Chang-Zhu-Tan & Chansha, Zhuzhou, Xiangtan & Mid-Yangtze River \\
\hline & Chang-Jiu & Nanchang, Jiujiang & Mid-Yangtze River \\
\hline & He-Huai & Hefei, Huainan & Central Anhui \\
\hline & Wu-Ma & Wuhu, Maanshan & Central Anhui \\
\hline \multirow{8}{*}{ Western region } & Xi-Xian & Xi'an, Xianyang & Guanzhong cities \\
\hline & Cheng-De & Chengdu, Deyang & Chongqing-Chengdu \\
\hline & Southern Guangxi & Nanning, Beihai, Qinzhou, Fangchenggang & Southern Guangxi \\
\hline & Gui-An & Guizhou, Anshun & Central Guizhou \\
\hline & Lan-Bai & Lanzhou, Baiyin & Lanzhou-Xining \\
\hline & Jiu-Jia & Jiuquan, Jiayuguan & - \\
\hline & Wu-Chang & Urumchi, Changji & Tianshan Mountains \\
\hline & Kui-Du-Wu & Kuytun, Dushanzi, Wusu & Tianshan Mountains \\
\hline \multirow{4}{*}{ Northeast region } & Shen-Fu & Shenyang, Fushun & Liaodong peninsula \\
\hline & Chang-Ji & Changchun, Jilin & Harbin-Changchun \\
\hline & Da-An & Daqing, Anda & Harbin-Changchun \\
\hline & Yan-Long-Tu & Yanji, Longjin, Tumen & 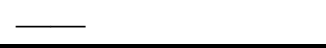 \\
\hline
\end{tabular}

*Quoted from Spatial organization Structure, patterns and dynamic mechannism of uni-cities in china, submitted to Acta Geographica Sinica. 


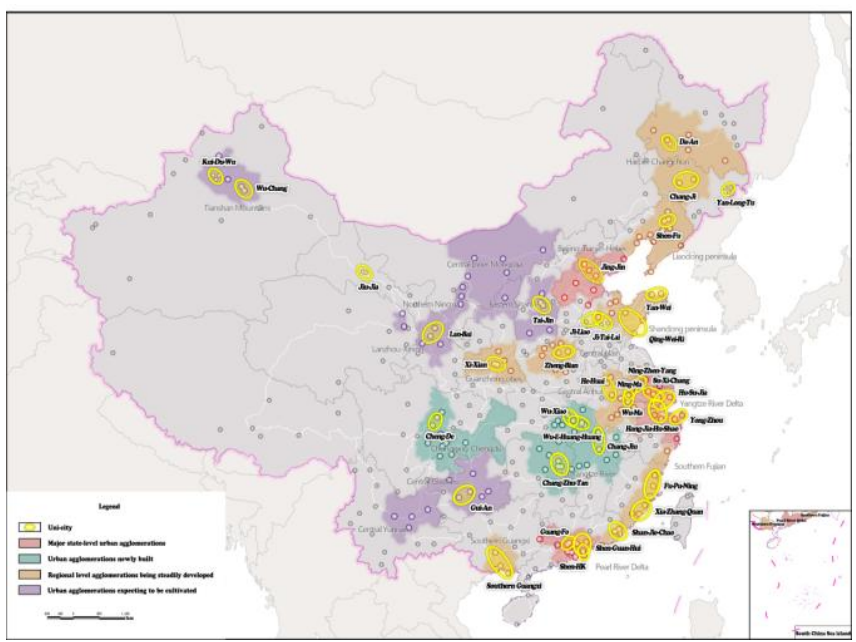

Figure 1. The " $17+8+8+4$ " spatial distribution pattern of unicities in China (quoted from Spatial organization Structure, patterns and dynamic mechannism of uni-cities in china, submitted to Acta Geographica Sinica)

\section{PROBLEMS OF FORMATION AND} DEVELOPMENT OF UNI-CITIES IN CHINA

\subsection{The identification standard is lacking and required to be improved}

There is no identification standard of uni-city at present, which leads to disordered clannishness regardless of the city's development condition and overestimation of their development expectation. Some cities even extend the scope of urban integration at random which is actually a manner of euphemistical regional privation. The spatial scope of uni-city is different from urban agglomeration. The formation and development of the later needs certain population size and economic magnitude (Fang, 2011), while the former is more focused on their mobility and interaction. Thus the substandard urban agglomeration may be qualified uni-city. As far as the distance is concerned, it is deemed that the cities should be adjacent.But as to the extent of proximity there is no uniform answer, of which" 30 kilometer" is the major number of the first batch of uni-city's distance. With the innovation of means of transportation and rapidity of vehicles, the limitation of spatial distance becomes weaken and time distance is still a significant constraint for urban integration.

\subsection{Land resource is utilized extensively while development quality is required to be improved}

Land resource is utilized extensively during the implementation of urban integration. Since physical space is easy to operate, the construction of new district, with which urban land has been substantially enlarged, is regarded as key to promote urban integration. Four of nine state-level new district approved by the State Council: Lanzhou new area, Xixian new area, Guian new area and the west coast of Qingdao new area, which are the important fulcrums for Lan-Bai integration, Xi-Xian integration, Gui-An integration and Qing-Wei-Ri integration respectively, are located in the main connection direction separated from the main city and occupy massive land resource to meet the requirement of elasticity of urban structure. Besides, since the secondary central city seeks rapid development and usually could provide less costly construction land, almost every city ajacent to metropolis proposes to build the co-construction industrial districts to undertake industrial transfer from the metropolis of the uni-city. Facing high land pressure and tough land authority, these new areas and industrial districts have to confront the common propositions to make transformation and increase efficiency accordingly.

\subsection{The collaboration is limited while top layer designs are required to be reinforced}

\subsubsection{Limitations of collaborative content}

Openning inter-city transportation, co-building industrial district, unifying area code and lowering bank charge, et al. are the "general action" in unicities. Generally speaking, the practices of urban integration remain in these facile physical forms, while industrial cooperation, technological innovation, environmental governance as well as cooperation of public service such as education, health care, employment, social security, which are related to the quality of urban integration still lag behind, let alone exploration on economic benefits distribution mechanism, household registration system, land system and social management(Wei et al.,2014).This reflects the issue, "evading the important questions for the easy" during urban integration to some extent. Innovating the governing manner, social construction and institutional arrangement to break administrative barriers will be domain directions for urban integration.

\subsubsection{Limitations of influenced region}

The present urban integration in our country are mainly integration between cities and reallocation of urban function. Existing integration plannings are focused on spatial organization of urban areas as well, ignoring spatial interaction of different level or in different scale(Chen,2012). The coordination with the hinterland and issues that how urban integration promotes rural development, urban-rural integration as well as nearby employment of transferring agricultural workers in the region are the ones which should be noted. Promoting transformation of the influenced area from "a corner" to "all-scale" will be domain perspective of urban integration.

\subsubsection{Limitations of coordinating approach}

Urban integration in China is still too dependent on the performance of governments, and its 
implementation process is closely related to local government's participation (Wang et al., 2009), lacking of widening participation of citizens, enterprises and NGOs. The existing coordinating patterns, no matter they are cross-administrative council, negotiation between heads of local government, branch organ of superior government or united party committee, et al. (Jiao \& Sun, 2011) always embody the vision and promise of local leaders. Urban integration plannings are definitive blueprint which are hard to accommodate dynamic change or market operation. Promoting transformation of coordinating approach from "single decision-making" to "multi-body participation" should be the main mechanism of urban integration.

\section{GOVERNANCE COUNTERMEASURES OF DEVELOPMENT OF UNI-CITIES IN CHINA}

To ensure urban sustainable development as well as healthy and orderly evolution to urban agglomerations, some policy countermeasures and suggestions for the construction of uni-cities are put forward as follows.

Make better use of urban planning for regional rescaling. As the most basic platform for distribution of policy resources, plannings, such as urban integration planning and new area planning are transforming more and more land to districts with lacation advantage and policy resource. These districts could concentrate preponderant resources to acquire urban competitiveness, which may be used as a approach for seeking authorization of construction land on the other hand (Liu \& Jin, 2012). So it is necessary to make better use of urban planning, properly construct the new spatial scales for governance of our country. Thus, as to the cities which are too distant and restricted by stage of development, it is more appropriate to progressively conduct spatial integration strategy and avoid extensive construction.

Further deepen collaborative contents of urban integration. The contents should be developed from the present spatial collaboration to industrial collaboration and social collaboration. Industry transition and industrial upgrading should be grasped to promote the indepth collaboration of uni-cities. In addition, the development concept such as green, smart and humanities should be combined in the process of urban integration.

Upgrade the competitiveness of central city. It is necessary to build international metropolis and regional central city which usually dominates the development of the uni-city and even the region, and rely on which the region could be embed in global urban system. Meanwhile, satellite cities and small cities ought to be developed with guidance as well, and promoted to embed in territorial urban system and gain development opportunities to form a networked, efficient, mobility and dynamic regional structure.

Establish and improve the institution of collaboration and organization of uni-cities. It's necessary to establish a specific coordinating institution on account of regional issues for governance of metropolitan area (Zhang et al., 2006). So it may be necessary to establish a development committee in uni-city to integrate competitive behavior and decide public issues with externality. What's more, market institutions should be regarded as a leading role in the process of urban integration. Citizens, NGO, specialists, scholars and other related-interest parties should be allowed to involve in the planning and conversation.

Establish information sharing mechanism as well as co-planning and co-review mechanism. Basic databases ought to be established and shared between uni-cities, and the planning information base ought to be updated dynamically and shared as well. It is also necessary to establish the notification and filing mechanism of adjacent belt planing, and the three plannings, urban planning, territorial planning, and social and economic development planning of the adjacent cities should be coordinated all in all.

\section{REFERENCES}

[1] Chen, X.L.et al. 2012. Research on implementation process of city-integration based on power restrict: a case study of Guangzhou-Foshan integration. Modern Urban Research, (07):64-69.

[2] Fang,C.L. 2011. New structure and new trend of formation and development of urban agglomarations in China. Geographical Science, 31(9):1025-1034.

[3] Ge,Y.et al. 2012. Research on regional spatial development strategy:a case study of integrative plan of Chang-Ji region. Shanghai Urban Planning Review, (4): 30-36.

[4] Jiao,Z. \& Sun,J. 2011. Research on China's urban cohesion mode and system design. Modern Urban Research, (06): 7 10.

[5] Li,K.P. et al. 2010. Research and practice of planning collaboration in urban cluster area: the example of guangzhou-Foshan urban integration. Planners, 26(9): 47 52.

[6] Liu, J.H. \& Jin,X. 2012. Large scale new area planning under national strategy. Diversity and inclusion: annual national planning conference. Kunming: Science and Technology Publishing Press.2012: 636-645.

[7] Song,Y. 2008.The implemention review o nintegration strategy and case study in China. Shanghai: Tongji University.

[8] Wang,D.et al. 2009. A review on the implement of city integration strategy. Urban Planning Forum, (04):74-78.

[9] Wei,Z.C.et al. 2014. Considerations on urban integration in China: take Guang-Fo integration for example. Urban Planning Forum, (2):80-86.

[10]Xin,M. 2007. Thoughts over integration of ShenyangFushun metropolitan area. City Planning Review, 31(10): 52-56.

[11]Zhang,J.X.et al. New trend of city agglomeration regions' development and planning. Planning's decade: annual national planning conference. Beijing: Urban Planning Society of China.2006:154-158. 